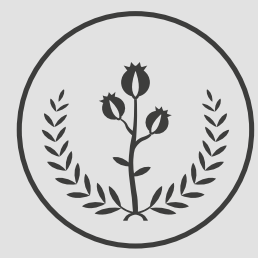

FUCS $-x$

$N^{0} 1.2021$

\title{
Complicaciones cardiovasculares en relación con la programación fetal
}

\author{
Nathalia Andrea Cerón MD \\ Oscar Octalivar Gutiérrez MD $^{\text {b }}$ \\ Oscar Mauricio Cerón MD \\ Robert Alirio Ortiz MD ${ }^{\mathrm{d}}$
}

\section{Cardiovascular complications related to fetal programming}

${ }^{a}$ Medicina y Cirugía. Universidad del Cauca. Popayán, Colombia.

${ }^{b}$ Ginecología y Obstetricia. Universidad del Cauca, Popayán, Colombia.

${ }^{c}$ Medicina, Universidad de Ciencias Aplicadas y Ambientales UDCA. Bogotá, Colombia.

${ }^{d}$ Ginecología y Obstetricia, Epidemiologí, Universidad del Cauca, Popayán, Colombia.

\section{RES U M EN}

Introducción: la programación fetal ofrece nuevas perspectivas sobre el origen de las enfermedades cardiovasculares, relacionando su aparición con factores perinatales. Objetivo: exponer evidencia que vincule las alteraciones gestacionales con las enfermedades cardiovasculares en la vida adulta del feto. Metodología: búsqueda en las bases de datos EBSCO, COCHRANE, MEDLINE, PROQUEST y SciELO de los artículos de revisión e investigaciones originales en inglés publicados en los últimos diez años. Se utilizaron términos MeSH para búsqueda controlada y se evaluaron los estudios con STROBE y PRISMA según correspondía. Resultados: los hallazgos sugieren que nacer con menos de $2600 \mathrm{k}$ guarda relación con diabetes mellitus (OR de 1.607 IC $95 \%$ 1.324-1.951), hipertensión arterial (OR de 1.15 IC 95\% 1.043-1.288) y menor función endotelial (1.94+0.37 vs $2.68+0.41$, p: 0.0001 ) en la adultez. La prematuridad se asocia con mayores presiones arteriales sistólicas (4.2 mmHg IC $95 \%$; 2.8 - 5.7 p 0.001) y diastólicas (2.6 mmHg IC 95\%; 1.2-4.0; p 0.001). Las alteraciones nutricionales maternas y la diabetes gestacional aumentan el riesgo de síndrome metabólico (OR 1.2 IC 95\% 0.9-1.7) y sobrepeso en la edad escolar (OR 1.81 IC $95 \% 1.18$ - 2.86). Conclusión: los resultados adversos en la gestación están relacionados con el desarrollo de enfermedades cardiovasculares en la vida adulta del feto expuesto.

Palabras clave: desarrollo fetal; enfermedades cardiovasculares; diabetes gestacional; trabajo de parto prematuro.

(C) 2021 Fundación Universitaria de Ciencias de la Salud - FUCS. Este es un artículo Open Access bajo la licencia CC BY-NC-ND (http://creativecommons.org/licenses/by-nc-nd/4.0/).

INFORMACIÓN DEL ARTÍCULO

Historia del artículo: Fecha recibido: enero 22 de 2019 Fecha aceptado: julio 23 de 2019
Autor para correspondencia. Dra. Nathalia Andrea Cerón nathaliaceron@unicauca.edu.co
DOI

10.31260/RepertMedCir.01217273.943 


\section{A BSTRACT}

Introduction: fetal programming offers new perspectives on the origin of cardiovascular diseases, relating their appearance with perinatal factors. Objective: to show the evidence associating gestational alterations with cardiovascular diseases in the offspring in adult life. Methodology: an EBSCO, COCHRANE, MEDLINE, PROQUEST and SciELO databases search of original review and research articles published in English in the last ten years was conducted. MeSH terms were used to perform a controlled search. The studies were analyzed accordingly using the STROBE and PRISMA reporting guidelines. Results: The findings suggest that a birth weight of less than $2600 \mathrm{~kg}$ is related with diabetes mellitus (OR $=1.607,95 \% \mathrm{CI} 1.324$ to 1.951$)$, hypertension (OR $=1.15,95 \%$ CI 1.043 to 1.288$)$ and impaired endothelial function $(1.94+0.37$ vs $2.68+0.41$, p: 0.0001 ) in adulthood. Prematurity is related with higher systolic blood pressure (4.2 mmHg 95\% CI; 2.8 to $5.7 \mathrm{p} 0.001$ ) and diastolic blood pressure $(2.6 \mathrm{mmHg} 95 \% \mathrm{CI} ; 1.2$ to $4.0 ; \mathrm{p} 0.001)$. Maternal nutritional alterations and gestational diabetes increase the risk of metabolic syndrome (OR $=1.295 \%$ CI 0.9 to 1.7) and overweight in school-age (OR $=1.8195 \%$ CI 1.18 to 2.86). Conclusion: adverse results during pregnancy are related with the development of cardiovascular diseases in the exposed fetus in adult life.

Key words: fetal development; cardiovascular disease; gestational diabetes; premature labor.

(C) 2021 Fundación Universitaria de Ciencias de la Salud - FUCS. This is an open access article under the CC BY-NC-ND license (http://creativecommons.org/licenses/by-nc-nd/4.0/).

\section{IN TRODUCCIÓN}

Las enfermedades cardiovasculares (ECV) son la principales causas de muerte a nivel mundial, con más de 17.3 millones al año, número que se espera superior a 23.6 millones en 2030. En el 2013 las muertes cardiovasculares representaron $31 \%$ de las mundiales, $80 \%$ ocurridas en países de bajos y medianos ingresos. ${ }^{1}$

Por otro lado, las alteraciones metabólicas como la resistencia a la insulina han determinado un riesgo adicional para la mortalidad cardiovascular. Se estima que en el mundo 347 millones de personas tenían diabetes en 2013 y se calcula que en 2012 fallecieron 1.5 millones de personas por esta causa. En la actualidad las proyecciones de la OMS definen la diabetes como posible séptima causa de mortalidad en $2030 .^{2}$

El gran impacto representado por estas patologías lo convierte en un tema central para la comunidad científica en busca de estrategias encaminadas a la disminución de la morbimortalidad. Es por eso que se hace necesario ahondar el conocimiento en la fisiopatología de estas entidades, dilucidando nuevos factores de riesgo para intervenir en la población.

Hace cerca de 30 años David James Barker, fisiólogo y epidemiólogo británico, propuso un cambio revolucionario en la historia natural de las ECV, al otorgar a la alteración del desarrollo normal en la vida intrauterina un papel fundamental como factor desencadenante de enfermedades crónicas no transmisibles. Reveló la existencia de una relación inversa entre el peso al nacer y la presión sistólica (PAS) en la vida adulta, que vincula un mayor riesgo de padecer ECV. ${ }^{3}$ Lo anterior lo asoció con un aumento significativo de la mortalidad coronaria en pacientes con bajo peso al año de edad, donde el autor concluye que la enfermedad coronaria está ligada con patrones específicos de crecimiento fetal desproporcionado, que son el resultado de la desnutrición fetal gestacional. ${ }^{4}$ Hoy se reconoce el desarrollo prenatal como un período crítico en la etiología de las enfermedades humanas ${ }^{5}$

La programación fetal (PF) se presenta tras la interrupción del desarrollo normal del feto por una noxa en un punto crítico de la vida intrauterina, que altera la expresión genética e involucra el desarrollo de enfermedades en la vida adulta. ${ }^{6}$ Es una reacción adaptativa al ambiente hostil que genera cambios epigenéticos (metilación, acetilación de histonas), reflejados en modificaciones fenotípicas permanentes. ${ }^{7,8} \mathrm{El}$ factor ambiental influencia al gen, ya que estados proinfamatorios, producen un impacto adverso sobre el desarrollo embrionario, al deteriorar la supervivencia celular y el crecimiento fetal, incluso a nivel placentario., ${ }^{9,10}$

Es así como se ha demostrado la relación entre diversas alteraciones obstétricas como bajo peso al nacer (BPN), parto prematuro, diabetes gestacional, alteraciones nutricionales y restricción intrauterina del peso (RCIU) con la aparición de ECV y metabólicas en la vida adulta del producto de la gestación expuesta. ${ }^{11}$

La presente revisión de literatura tiene como objetivo exponer la evidencia que enlaza las diversas alteraciones obstétricas con el desarrollo de ECV por medio de la PF.

\section{METODOLOGÍA}

Se realizó una búsqueda en la literatura mundial en diferentes bases de datos EBSCO, COCHRANE, MEDLINE, PROQUEST y SciELO, seleccionando artículos originales en 
inglés publicados en los últimos diez años, según información disponible. Se utilizaron términos $\mathrm{MeSH}$ "fetal programing, hypertensive disorders, lowbirthweight, pretermbirth, gestational diabetes, diabetes mellitus, arterial hypertension, obesity, overweight, dyslipidemia, cardiovascular disease, coronaryheartdisease, cardiovascular mortality".

Se seleccionaron artículos de revisión e investigaciones originales que determinen y expliquen la relación entre adultos expuestos a parto prematuro, BPN, alteraciones nutricionales maternas durante la gestación y diabetes gestacional, con el desarrollo de dislipidemias, diabetes mellitus e hipertensión arterial. La calidad de los estudios fue evaluada con STROBE para estudios observacionales y PRISMA para revisiones sistemáticas, ${ }^{12,13}$ obteniendo un total de 40 artículos, de los cuales tres son revisiones sistemáticas, una con meta-análisis, siete estudios de cohorte y cuatro análisis de casos y controles. Se incluyeron dos guías epidemiológicas y revisiones de literatura. Como limitación se presentaron artículos de calidad publicados fuera del rango estipulado.

\section{RES U LTA D OS}

En una cohorte mixta se estudiaron la relación entre el peso al nacer y el riesgo de dislipidemias en 3603 hombres y 3583 mujeres entre 44 y 45 años, se examinaron datos sobre el peso al nacer y se compararon con niveles de colesterol total, LDL y HDL en mmol/L. Descubrieron que el incremento de $1 \mathrm{k}$ al nacer en mujeres se relaciona con disminución estadística significativa en los niveles de colesterol total (- $0.13 \mathrm{mmOl} / \mathrm{L}), \mathrm{LDL}(-0.07 \mathrm{mmOl} / \mathrm{L})$ y triglicéridos ($0.09 \mathrm{mmOl} / \mathrm{L}$ ), sin afectar los niveles de HDL, mientras en hombres el incremento de $1 \mathrm{k}$ al nacer se relaciona solo con disminución de los niveles de triglicéridos (- $0.06 \mathrm{mmOl} /$ L). ${ }^{14}$ Por otro lado, en un metaanálisis afirmaron que la disminución de $1.39 \mathrm{mg} / \mathrm{dL}(-0.036 \mathrm{mmol} / \mathrm{L})$ de colesterol libre se relaciona con el incremento de $1 \mathrm{k}$ al nacer (IC 95\%1.81-0.97 mg/dL). ${ }^{15}$ Concluyen que existe una asociación inversa entre el peso al nacer y los triglicéridos en ambos sexos.

En una revisión sistemática realizada por Whincup y col. 2008 se evidenció que el incremento de $1 \mathrm{k}$ en el peso al nacer se relaciona con menor riesgo de diabetes tipo 2 (OR 0.78 IC 95\% 0.72-0.84). ${ }^{16}$ Además, independiente del sexo, el índice de masa corporal (IMC), historia de diabetes paterna o materna, estatus socioeconómico y estilo de vida, el aumento de $1 \mathrm{k}$ al nacer se relaciona con una disminución en el riesgo de diabetes tipo 2 (OR 0.49 IC 95\% $0.35-$ 0.69). ${ }^{17}$ Lo anterior permite inferir que el peso al nacer está inversamente relacionado con el riesgo de diabetes tipo 2.

La relación entre BPN y mortalidad cardiovascular fue evaluado por Wei y col. en 2007, en una cohorte donde se estudiaron 81538 niños entre 6-18 años y la asociación entre su peso al nacer con obesidad, diabetes mellitus, hipertensión arterial e hipercolesterolemia. Se evidenció que el riesgo de obesidad se asocia con alto peso al nacer $>$ $4000 \mathrm{~g}$ (OR 1.672 IC 95\% 1.514-1.846). El riesgo de diabetes se vinculó con un bajo y alto peso al nacer, la curva tiene forma de U, entre los nacidos con menos de $2600 \mathrm{~g}$ (OR 1.607 IC $95 \% 1.324-1.951)$ y más de 4000 g (OR 1.661 IC 95\% 1.401 - 1.971). El riesgo de hipertensión se asocia con un peso al nacer menor de 2600 g (OR 1.159 IC 95\% 1.0431.28). ${ }^{18}$

Las alteraciones nutricionales maternas durante la gestación se explicaron en dos estudios. De Rooij y col. (2007) siguieron la descendencia de 783 mujeres sometidas a hambruna prenatal, afirmando que la exposición a ella implicó un aumento significativo de riesgo para síndrome metabólico (OR 1.2 IC 95\% 0.9-1.7) y obesidad. ${ }^{19}$ Además, tenían concentraciones de triglicéridos significativas más altas (0.1 g/L; $0.2 \mathrm{~g} / \mathrm{L})$ y más bajas de HDL (-0.08 mmol / $\mathrm{L} ;-0.14 \mathrm{mmol} / \mathrm{L})$ en especial en hombres. ${ }^{20}$ Bettiol y col. (2007) determinaron las consecuencias de la RCIU sobre el IMC en 519 niños a la edad de 8 y 18 años, estableciendo que aquellos que tuvieron restricción y sobrepeso en la edad escolar primaria, mostraban un efecto positivo y fuerte sobre el IMC a los 18 años de edad (coeficiente 5.03 IC $95 \%$ 4.27-5.79) generando un aumento en su IMC de $6.90 \mathrm{k} / \mathrm{m} 2$ (IC 95\% 4.55-9.26). ${ }^{21}$

Baptiste y col. (2012) valoraron el nexo de la diabetes gestacional y el crecimiento infantil a los 3, 4 y 7 años en una cohorte de 28358 binomios, madres e hijos. Concluyeron que al tener en cuenta la edad e IMC materno, ganancia de peso en el embarazo, historia familiar de diabetes, peso al nacer y haber desarrollado diabetes gestacional, implica una mayor probabilidad de sobrepeso a las edades de 4 (OR 1.81 IC $95 \% 1.18-2.86$ ) y 7 años (OR 1.61 IC 95\% 1.07-1.28). ${ }^{22}$

El evento de mayor correlación fue la presión arterial en la vida adulta con el antecedente de nacer prematuro. Parkinson y col. (2013) evaluaron 27 estudios comparando 17030 adultos nacidos prematuros y 295261 adultos nacidos a término, concluyendo que la prematurez se asocia con un incremento significativo PAS de $4.2 \mathrm{~mm} \mathrm{Hg}$ (IC 95\% 2.85.7 P 0.001), y un aumento en la presión arterial diastólica (PAD) de $2.6 \mathrm{~mm} \mathrm{Hg}$ (IC 95\% 1.2-4.0 P 0.001). En cuanto al género encontraron un aumento de $2 \mathrm{~mm} \mathrm{Hg}$ en la PAS en la vida adulta en hombres (IC 95\% 0.5-3.5 P 0.007) y en mujeres de $4.9 \mathrm{~mm} \mathrm{Hg}$ (IC 95\% 3.3-6.6 P 0.001), así como aumento en la PAD en hombres de $1.3 \mathrm{~mm} \mathrm{Hg}$ (IC 95\% 0.1$2.4 \mathrm{P} 0.03$ ) y en mujeres de $2.9 \mathrm{~mm} \mathrm{Hg}$ (IC 95\% 1.6- 4.1 $\mathrm{P}$ 0.06); concluyeron que los adultos con antecedente de nacer pretérmino presentaron mayores cifras de PAS y PAD, independiente del sexo. ${ }^{23}$

Dalziel y col. 2007 en una cohorte prospectiva de 458 pacientes de 30 años, analizaron la edad gestacional al nacer con factores de riesgo cardiovascular como PAS y tolerancia a la glucosa. Se encontró que los prematuros tienen un 
incremento significativo de $3.5 \mathrm{~mm} \mathrm{Hg}$ (IC 95\% 0.9-6.1 mm $\mathrm{Hg}, \mathrm{P}: 0.009$ ) en la PAS y mayor resistencia a la insulina a los 30 años [registro (área de insulina bajo la curva) $=0.17$, 95\% IC 0.05-0.28 P 0.006]. ${ }^{24}$

Como factor esencial en la fisiopatología de la ECV está la calidad endotelial. Bassareo y col. en 2010 evaluaron el riesgo cardiovascular con el daño endotelial mediante pletismografía (flujo mediado por dilatación de la arteria braquial) a través de un estudio de casos y controles. Compararon un grupo de nacidos prematuros con extremo BPN por debajo de $1000 \mathrm{~g}$ con el control de nacidos a término con peso adecuado. Evidenciaron una función endotelial muy reducida en la población con antecedente de extremo bajo peso al nacer comparado con los controles $(1.94+0.37$ vs $2.68+0.41$ P 0.0001$)$. Además esta función se correlacionó bien con la edad gestacional ( $0.56 \mathrm{P}<0.0009)$ y el peso al nacer ( $0.63 \mathrm{P}<0.0001$ ), concluyendo que este último se relaciona inversamente con la función endotelial. ${ }^{25}$ Leeson y col. en 2001 evaluaron la respuesta vascular dependiente e independiente del endotelio mediante ultrasonido de alta definición en una cohorte de 315 adultos. Refieren que el BPN participa en la patogénesis de ateroesclerosis en la vida adulta al tener una menor respuesta endotelial a la dilatación mediada por flujo (coeficiente: $0.18 \mathrm{~kg}-1$ IC $95 \%$ $0.004-0.35, \mathrm{P}=0.04) .{ }^{26}$

\section{IS C USIÓN}

La PF tiene lugar cuando el entorno óptimo en el que crece el feto se ve interrumpido por factores hostiles, en especial durante períodos críticos de la organogénesis. Funciona como un mecanismo importante que favorece la homeostasis del organismo en condiciones inadecuadas. Estudios exhaustivos indican que los eventos de la vida temprana juegan un papel poderoso al influir en la posterior susceptibilidad a ciertas enfermedades crónicas, lo anterior sustentado en una hipótesis conocida como "origen fetal de la enfermedad" que propone que la hipertensión, la resistencia a la insulina y la dislipidemia en la edad adulta se originan a través de la adaptación fetal al medio intrauterino adverso. De los eventos obstétricos más representativos se ha encontrado evidencia como participantes de la PF: BPN, parto prematuro, diabetes gestacional, y alteraciones nutricionales maternas durante el embarazo y la restricción del crecimiento intrauterino (RCIU). ${ }^{27}$

En primera instancia, el adecuado peso al nacer tiene influyentes como son el nacimiento a término, el crecimiento y la nutrición materna adecuada. Se ha establecido una relación inversa entre el peso al nacer con la fisiología de los lípidos y la glucosa, ${ }^{14,17}$ siendo los valores extremos los causales fetales más relacionados con dislipidemia, obesidad, resistencia a la insulina e hipertensión arterial. ${ }^{18}$ Se ha encontrado que la variación en los genes KCNJ11, BDNF, PFKP, PTER y SEC16B en pacientes pequeños para la edad gestacional se relaciona con el desarrollo de diabetes mellitus y obesidad, ${ }^{28}$ incluso con una mayor predisposición a la ingesta de grasas, constituyendo un factor adicional para la obesidad sobre todo en aquellos con peso menor 2600 g. ${ }^{29}$ Además, parece que la prematuridad influye en el riesgo de diabetes tipo 2 mediante sensibilidad insulínica atenuada, junto con efectos combinados sobre la función de las células beta. ${ }^{24}$

El curso de la gestación es determinante clave, pues la nutrición materna y la función placentaria pueden influir en el desarrollo del feto y el nacimiento, en especial en el síndrome metabólico. ${ }^{30,31}$ La desnutrición o la obesidad materna gestacional podrían programar la resistencia a la insulina, ${ }^{32}$ favoreciendo mecanismos reguladores para el almacenamiento de energía después del nacimiento, a pesar de la disponibilidad adecuada o excesiva de nutrientes lo que resulta en diversos trastornos metabólicos, fenómeno conocido como la hipótesis del fenotipo ahorrativo. ${ }^{33} \mathrm{Si}$ bien la gestación es una situación diabetogénica en sí misma, es probable que más del 10\% de las embarazadas desarrollen diabetes gestacional, ${ }^{32}$ que se refleja en la descendencia como una mayor tendencia al sobrepeso u obesidad en la infancia. $^{22}$ El riesgo está definido debido a la acción de la adiponectina y la leptina, dos hormonas sensibilizantes a la insulina, antiaterogénicas y antiinflamatorias, disminuidas por una disregulación debida a la diabetes gestacional o estrés materno, entre otros efectos específicos en los transportadores de glucosa. ${ }^{34}$

En cuanto a la presión arterial en la vida adulta, el antecedente de nacer prematuro fue el evento de mayor correlación, reportando un incremento significativo PAS de $4.2 \mathrm{~mm} \mathrm{Hg}$, y un aumento en la PAD de $2.6 \mathrm{~mm}$ $\mathrm{Hg}^{23}$ Etiológicamente se atribuye a la PF provocar la disminución del número de nefronas, la disfunción vascular y el desequilibrio entre factores vasoactivos potentes, que programa un aumento en la resistencia periférica total. ${ }^{6}$ Estudios en animales sometidos a restricción de nutrientes concluyeron que las consecuencias dependen del momento en que se programa el procedimiento. Por ejemplo, si se presenta durante el periodo nefrogénico puede generar una reducción en el número de nefronas, por ende un aumento de la presión arterial de cerca de $13 \mathrm{~mm} \mathrm{Hg}$. Dada la importancia de los riñones en la regulación a largo plazo de la presión arterial, estos estudios sugieren que una agresión durante el desarrollo renal conlleva cambios en la regulación de la presión arterial en el futuro. ${ }^{35,36}$

De igual forma, la hipertensión está influenciada en forma directa por la función endotelial, el sistema renina angiotensina y el óxido nítrico. Existe una correlación entre la presión arterial sistólica elevada en la adolescencia y la disfunción de las células endoteliales mediada por un aumento de la rigidez arterial, ${ }^{37}$ una característica común en la hipertensión esencial debido a una disminución del contenido de elastina y de la distensibilidad arterial en sujetos nacidos prematuros, así como la alteración de la 
regulación de sustancias vasoactivas y vasodilatadoras. ${ }^{38}$

Las células endoteliales participan en la homeostasis vascular e inflamatoria sujetas a influencias mecánicas y metabólicas, es por eso que puede usarse como indicador del sistema cardiovascular afectado en alteraciones del peso al nacer. ${ }^{25,39}$ En la literatura, se describe su etiología basada en el incremento en la formación de procesos trombogénicos y ateroscleróticos. ${ }^{40}$

La evidencia presenta variedad de estudios tanto epidemiológicos como experimentales ya sea en humanos o animales, que han demostrado la importancia del curso del embarazo en la historia natural de la enfermedad cardiovascular y metabólica. Independiente del factor anómalo en la gestación ya sea materno (preeclampsia, diabetes gestacional o enfermedad inflamatoria) o fetal y neonatal (restricción del crecimiento intrauterino y nacimiento prematuro), es congruente establecer un mayor riesgo de enfermedad crónica para la vida adulta en fetos expuestos.

\section{CONCLUSIÓN}

La gestación es una ventana clave de sensibilidad debido a las primeras interacciones entre el feto y el medio ambiente, con consecuencias de por vida. Es claro que durante este período las capacidades funcionales y reguladoras de los sistemas y órganos son predeterminadas a largo plazo a través de una adaptación bajo la influencia del entorno, que se condiciona con el riesgo de enfermedades crónicas posteriores, como hipertensión, obesidad, diabetes tipo 2 y otras enfermedades no transmisibles en la edad adulta.

\section{CONFLICTO DE INTERÉS}

Los autores declaran no tener ningún conflicto de intereses.

\section{FINA N C I AC I ÓN}

El presente artículo no recibió financiación alguna.

\section{REFEREN CIAS}

1. Mozaffarian D, Benjamin EJ, Go AS, Arnett DK, Blaha MJ, Cushman M, et al. Heart Disease and Stroke Statistics-2016 Update: A Report From the American Heart Association. Circulation. 2016;133(4):e38-360.

2. Ministerio de Salud y protección Social, Dirección de Epidemiología y Demografía. Análisis de Situación de Salud. Colombia, 2016. Colombia: Ministerio de Salud y protección Social; 2015. p. 149.
3. Barker DJ, Osmond C, Golding J, Kuh D, Wadsworth ME. Growth in utero, blood pressure in childhood and adult life, and mortality from cardiovascular disease. BMJ. 1989;298(6673):564-7.

4. Barker DJ. Fetal origins of coronary heart disease. Br Heart J. 1993;69(3):195-6.

5. Marciniak A, Patro-Malysza J, Kimber-Trojnar Z, Marciniak B, Oleszczuk J, Leszczynska-Gorzelak B. Fetal programming of the metabolic syndrome. Taiwan J Obstet Gynecol. 2017;56(2):133-8.

6. Perrone S, Santacroce A, Picardi A, Buonocore G. Fetal programming and early identification of newborns at high risk of free radical-mediated diseases. World J Clin Pediatr. 2016;5(2):17281.

7. Salam RA, Das JK, Bhutta ZA. Impact of intrauterine growth restriction on long-term health. Curr Opin Clin Nutr Metab Care. 2014; 17(3):249-54.

8. Gluckman PD, Hanson MA, Cooper C, Thornburg KL. Effect of in utero and early-life conditions on adult health and disease. New Engl J of Med. 2008;359(1):61-73.

9. Robertson SA, Chin PY, Femia JG, Brown HM. Embryotoxic cytokines-Potential roles in embryo loss and fetal programming. $\mathrm{J}$ Reprod Immunol. 2018;125:80-8.

10. Meister TA, Rexhaj E, Rimoldi SF, Scherrer U, Sartori C. Fetal programming and vascular dysfunction. Artery Res. 2018;21:6977.

11. Hsu P, Nanan R. Foetal immune programming: hormones, cytokines, microbes and regulatory T cells. J Reprod Immunol. 2014;104-105:2-7.

12. Vandenbroucke JP, Von Elm E, Altman DG, Gøtzsche PC, Mulrow $\mathrm{CD}$, Pocock SJ, et al. Mejorar la comunicación de estudios observacionales en epidemiología (STROBE): explicación y elaboración. Gac Sanit. 2009;23(2):158el-e28.

13. Urrútia G, Bonfill X. Declaración PRISMA: una propuesta para mejorar la publicación de revisiones sistemáticas y metaanálisis. Med Clin. 2010;135(11):507-11.

14. Cooper R, Power C. Sex differences in the associations between birthweight and lipid levels in middle-age: findings from the 1958 British birth cohort. Atherosclerosis. 2008;200(1):141-9. Epub 2008/01/01.

15. Illingworth RS. Birth weight and subsequent weight. Br Med J. 1950;1(4645):96.

16. Whincup PH, Kaye SJ, Owen CG, Huxley R, Cook DG, Anazawa S, et al. Birth weight and risk of type 2 diabetes: a systematic review. Jama. 2008;300(24):2886-97. Epub 2008/12/26.

17. Pilgaard K, Faerch K, Carstensen B, Poulsen P, Pisinger C, Pedersen $\mathrm{O}$, et al. Low birthweight and premature birth are both associated with type 2 diabetes in a random sample of middle-aged Danes. Diabetologia. 2010;53(12):2526-30. doi: 10.1007/s00125-010-1917-3.

18. Wei JN, Li HY, Sung FC, Lin CC, Chiang CC, Li CY, et al. Birth weight correlates differently with cardiovascular risk factors in youth. Obesity (Silver Spring). 2007;15(6):1609-16. doi: 10.1038/ oby.2007.190. doi: 10.1016/j.molmet.2018.06.009.

19. Beeson JH, Blackmore HL, Carr SK, Dearden L, Duque-Guimarães DE, Kusinski LC, et al. Maternal exercise intervention in obese pregnancy improves the cardiovascular health of the adult 
male offspring. Mol Metab. 2018;16:35-44. doi: 10.1016/j. molmet.2018.06.009

20. de Rooij SR, Painter RC, Holleman F, Bossuyt PM, Roseboom TJ. The metabolic syndrome in adults prenatally exposed to the Dutch famine. Am J Clin Nutr. 2007;86(4):1219-24. doi: 10.1093/ ajcn/86.4.1219

21. Bettiol H, Sabbag Filho D, Haeffner LS, Barbieri MA, Silva AA, Portela A, et al. Do intrauterine growth restriction and overweight at primary school age increase the risk of elevated body mass index in young adults?. J Med Biol Res. 2007;40(9):1237-43. doi: 10.1590/s0100-879x2007000900011

22. Baptiste-Roberts K, Nicholson WK, Wang NY, Brancati FL. Gestational diabetes and subsequent growth patterns of offspring: the National Collaborative Perinatal Project. Matern Child Health J. 2012;16(1):125-32. doi: 10.1007/s10995-011-0756-2

23. Parkinson JR, Hyde MJ, Gale C, Santhakumaran S, Modi N. Preterm birth and the metabolic syndrome in adult life: a systematic review and meta-analysis. Pediatrics. 2013;131(4):e1240-63. doi: 10.1542/ peds.2012-2177

24. Dalziel SR, Parag V, Rodgers A, Harding JE. Cardiovascular risk factors at age 30 following pre-term birth. Int J Epidemiol. 2007;36(4):907-15. doi: 10.1093/ije/dym067.

25. Bassareo PP, Fanos V, Puddu M, Demuru P, Cadeddu F, Balzarini M, et al. Reduced brachial flow-mediated vasodilation in young adult ex extremely low birth weight preterm: a condition predictive of increased cardiovascular risk? J Matern Neonatal Med. 2010;23 Suppl 3:121-4. Epub 2010/10/12.

26. Leeson CP, Kattenhorn M, Morley R, Lucas A, Deanfield JE. Impact of low birth weight and cardiovascular risk factors on endothelial function in early adult life. Circulation. 2001;103(9):1264-8. doi: 10.3109/14767058.2010.506811.

27. Hocher B. More than genes: the advanced fetal programming hypothesis. J Reprod Immunol. 2014;104-105:8-11. doi: 10.1016/j. jri.2014.03.001.

28. Morgan AR, Thompson JM, Murphy R, Black PN, Lam WJ, Ferguson LR, et al. Obesity and diabetes genes are associated with being born small for gestational age: results from the Auckland Birthweight Collaborative study. BMC Med Genet. 2010;11:125. doi: 10.1186/1471-2350-11-125.

29. Bischoff AR, Portella AK, Paquet C, Dalle Molle R, Faber A, Arora
$\mathrm{N}$, et al. Low birth weight is associated with increased fat intake in school-aged boys. Br J Nutr. 2018;119(11):1295-302.

30. Taniguchi K, Kawai T, Hata K. Placental Development and Nutritional Environment. Adv Exp Med Biol. 2018;1012:63-73. doi: 10.1007/978-981-10-5526-3_7.

31. Nolan CJ, Damm P, Prentki M. Type 2 diabetes across generations: from pathophysiology to prevention and management. Lancet. 2011;378(9786):169-81. doi: 10.1016/S0140-6736(11)60614-4.

32. Plagemann A. Maternal diabetes and perinatal programming. Early Hum Dev. 2011;87(11):743-7. doi:10.1016/j. earlhumdev.2011.08.018

33. Briana DD, Malamitsi-Puchner A. Intrauterine growth restriction and adult disease: the role of adipocytokines. European journal of endocrinology. 2009;160(3):337-47. doi: 10.1530/EJE-08-0621.

34. Ornoy A. Prenatal origin of obesity and their complications: Gestational diabetes, maternal overweight and the paradoxical effects of fetal growth restriction and macrosomia. Reprod Toxicol. 2011;32(2):205-12. doi: 10.1016/j.reprotox.2011.05.002.

35. Langley-Evans SC, Welham SJ, Jackson AA. Fetal exposure to a maternal low protein diet impairs nephrogenesis and promotes hypertension in the rat. Life Sci. 1999;64(11):965-74. doi: 10.1016/ s0024-3205(99)00022-3.

36. Ojeda NB, Grigore D, Alexander BT. Intrauterine growth restriction: fetal programming of hypertension and kidney disease. Adv Chronic Kidney Dis. 2008;15(2):101-6. doi: 10.1053/j. ackd.2008.01.001.

37. Van De Maele K, Devlieger R, Gies I. In utero programming and early detection of cardiovascular disease in the offspring of mothers with obesity. Atherosclerosis. 2018;275:182-95. doi: 10.1016/j.atherosclerosis.2018.06.016.

38. Simeoni U, Armengaud JB, Siddeek B, Tolsa JF. Perinatal Origins of Adult Disease. Neonatology. 2018;113(4):393-9. doi: 10.1159/000487618.

39. Balci MM, Acikel S, Akdemir R. Low birth weight and increased cardiovascular risk: fetal programming. Int J Cardiol. 2010;144(1):110-1. doi: 10.1016/j.ijcard.2008.12.111.

40. Ferreira VR, Jardim TV, Póvoa TR, Mendonça KL, Nascente FN, Carneiro CS, et al. Birth weight and its association with blood pressure and nutritional status in adolescents. J Pediatr. 2018;94(2):184-91. doi: 10.1016/j.jped.2017.04.007. 